\title{
Investigation and Prototype Design of Collaborative Virtual Learning Enivronments
}

\author{
Mohammad Saleem Darwaish and Fang Wang \\ Department of Information Systems and Computing \\ Brunel University \\ Uxbridge UB8 3PH, UK \\ e-mail: fang.wang@brunel.ac.uk
}

\begin{abstract}
Virtual learning environments (VLEs) are online learning systems that are used to enhance a student's learning experience by providing a set of teaching, learning and communication tools. The current VLEs, however, are not fully utilised or exploited to support effective collaborative learning. This paper investigates how to build effective online collaborative virtual learning environments, by exploring the requirements of VLEs from personal users. A prototype is then created to show how the designs of current VLEs can be improved to provide the essential functionalities and ease of use.

Keywords-online learning, virtual learning environment, collaborative learning, learning community
\end{abstract}

\section{INTRODUCTION}

Online learning has nowadays become an integral part of the formal learning process. Schools, colleges and universities, invest a lot of resources and effort to build efficient online learning environments but usually they focus more on creating and maintaining the system rather than improving the delivery of education. Numerous courses are offered by educational institutes, however, the quality of the courses provided online have become a concern [6] and the cost to create and maintain an online learning system has also become an issue [7].

A learning community is described to be an environment where people of similar interests and experience gather to learn together. An online learning community is one where electronic virtual learning environments (VLEs) are used to create and manage it [10]. Educational organisations are trying to develop online learning communities that can improve the learning experience and enhance the quality of teaching, by giving students the opportunity to engage and share ideas in virtual environments [8]. While current VLEs such as Blackboard, WebCT and COSE provide useful functionalities for online learning, they are less efficient in supporting collaborative learning of groups of learners. This paper investigates how to build effective online collaborative virtual learning environments. It explores the characters and requirements of collaborative virtual learning environments. A prototype is then created to design the essential functionalities and to show how the designs of current online learning platforms can be improved to provide ease of use.

The remaining of this paper is organised as follows. Section II introduces the background of collaborative virtual learning environments. User requirements for VLEs are investigated through questionnaires and the results are presented in Section III. According to the questionnaire results, Section IV describes our prototype design of improved virtual learning environments. The last section concludes the paper.

\section{BACKGROUND}

The advancement in multimedia and internet technologies has practically enhanced the applicability of collaborative learning via the Internet. It has been indicated that in 2006 over 3.5 million students in different educational institutions were involved in internet based collaborative learning within America alone [2] and there was a continuous increase in this number by 12 to $14 \%$ on a yearly basis [1, 3]. This fast evolving trend in collaborative learning may result in the perception of internet based collaborative learning as a foremost form of education delivery. The results of the Sloan survey by Allen \& Seaman showed that learners usually appeared to be, at the worst, satisfied with their engagement in Internet based lessons in a comparable fashion as to the way they were with regular learning approaches [2].

In his research, Thurston evaluated the effectiveness of the virtual learning environment created and utilised by University of Dundee to assist distance learning students enrolled in postgraduate programs [10]. Out of a sample of 47 students, 31 chose to receive learning support through the virtual learning environment while 16 chose to receive learning support via the existing techniques, e.g., telephone and email. The outcomes of the research showed that participants that actively engaged in online learning through the virtual learning environment reported an increased feeling of connectedness as being involved in a broader learning community. The results further showed that students exhibited a higher academic achievement levels than those who did not receive their learning through the VLE.

Current online learning platforms such as Blackboard and Moodle have offered useful virtual learning environments to help students access class content, grades, assessments, and other class tools online. They have also provided a social space where students and teachers can interact through threaded discussions or chat. These VLEs, however, as pointed out in $[4,5]$, lacked the necessary ingredients and interest, and the structure and content did not encourage regular use and participation. In order to provide effective VLEs, we need to acknowledge the requirements from real users and build suitable virtual learning environments according to the user needs. In the next section, we will introduce the investigation of user requirements. 


\section{USER REQUIREMENT INVESTIGATION}

\section{A. Questionnaire and Participants}

To investigate requirements from personal users, a questionnaire was produced to evaluate the features of the current VLEs and the functionalities desired by users. Questionnaire was utilised because it provides quick collection of information in a standardised way thus making the analysis process relatively straightforward.

The questionnaire consisted a combination of close ended questions (qualitative data) and open ended questions (quantitative data). The closed ended questions were used to evaluate the system functionality and the open ended questions were employed to acquire respondents' attitude and understanding of the system. The questionnaire was distributed to Computer Science and Information systems students in Brunel University as they are very well aware of the current VLE (Blackboard) utilised and its functionalities. Email was used to distribute the questionnaire to all the students in the department and in total 30 valid responses were received.

\section{B. Quantitative responses analysis}

There were eight questions designed in this section. The first five questions evaluated the usability of the current VLE system, such as Blackboard used at Brunel University. The other three questions examined the users' attitude to online communication, document sharing and storage, which are nowadays more often used for collaborative learning, particularly with the emergence of cloud computing.

The first four questions were:

Question 1: Please describe your experience to interact with the VLE system, e.g., while uploading or dowonloading files, e.g., assignment or lecture slides.

Question 2: Please describe your experience when using the system while performing a task, e.g., writing a discussion post.

Question 3: Please describe your experience about the usability of the system e.g., user friendly interface, easy to read font, colour, size.

Question 4: Please describe your experience when using the system while navigating through pages.

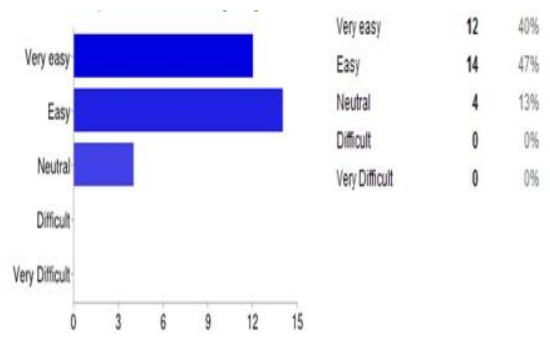

Figure 1 Responses to Question 1

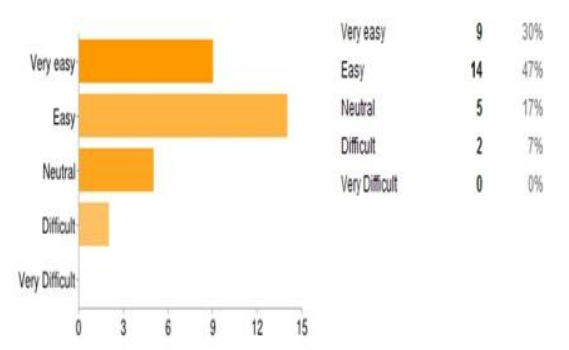

Figure 2 Responses to Question 2
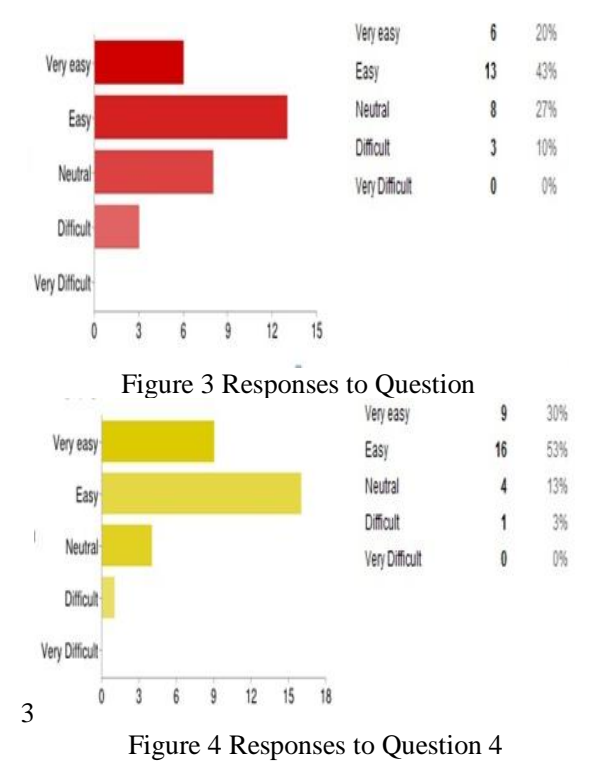

Figure 1 - Figure 4 show the voting answers of these four questions. The responses to these questions are very similar: the majority of the participants found it easy and a few less participants found it very easy to perform tasks in the current VLE. A small number of participants had neutral feelings and fewer or even no participants thought it difficult to perform the tasks.

Question 5: does the system contain any system error messages that clearly tell you how to fix them if any occurs?

Figure 5 demonstrates that most participants $(73 \%)$ are unaware of the manuals or help section of the system that can be used to deal with errors, in spite the fact that the help section link is right at the top of the screen of every page of the VLE. This meant that the help section required changing to make it more obvious.

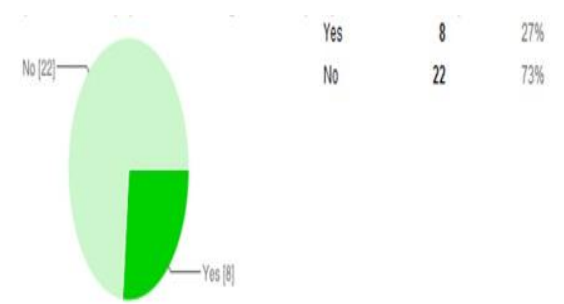

Figure 5 Responses to Question 5 
Question 6: How do you prefer to communicate with your tutor or fellow students?

Figure 6 illustrates most of the participants preferred email or instant messaging, while some preferred discussion and video conferencing.

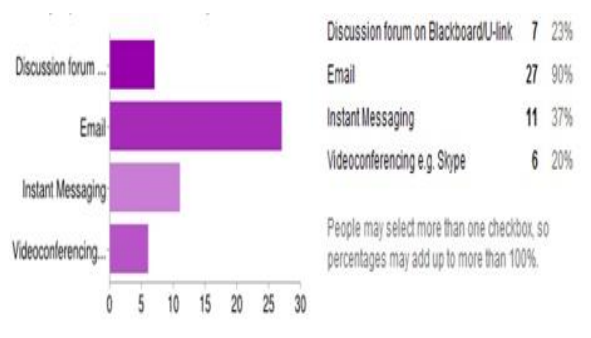

Figure 6 Responses to Question 6

Question 7: Would you use applications such as Spreadsheet and Word document provided online through a web browser, accessible anywhere for free instead of the desktop application such as Microsoft Excel and Microsoft Word?

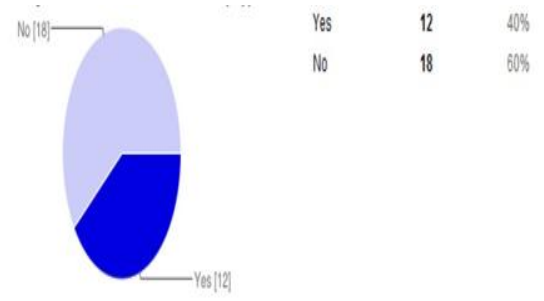

Figure 7 Responses to Question 7

Figure 7 shows $60 \%$ of the participants said No when they were asked whether they would use Cloud computing software such as Spreadsheet and Word and $40 \%$ that is 12 of the total respondents said that they would. The majority of the respondents prefer desktop application to cloud. This may be due to respondents' unfamiliarity with such technology. This is not surprising because most people had the same reaction when they first used Google Docs. These people, however, finally found the Google Docs to be a very flexible and efficient tool after the use.

Question 8: If you could store all your files online that can be accessible anywhere anytime like an online USB, would you use this feature?

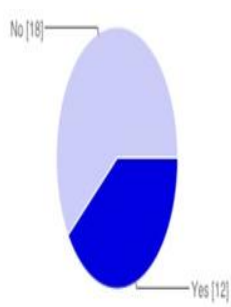

Figure 8 Responses to Question 8
Figure 8 demonstrates $87 \%$ of the respondents would use the file storage feature provided as a function of the Virtual learning environment. Actually a lot of students in Brunel already use some form of online storage such as Dropbox.

\section{Qualitative Reponses Analysis}

The qualitative responses for open questions were collected and examined manually. Patterns emerged from the data were then categorised and interpreted.

Question 9: When working in group, how useful is the system to collaborate with group members while communicating and performing a task?

Table 1 Responses to Question 9

\begin{tabular}{|cc|}
\hline Patterns/Categories & $\begin{array}{c}\text { No of } \\
\text { Respondents }\end{array}$ \\
\hline $\begin{array}{c}\text { Easy to share files among Group members } \\
\text { Discussion and wiki }\end{array}$ & 7 \\
\hline $\begin{array}{c}\text { Lack of consistency per module Causes } \\
\text { Confusion }\end{array}$ & 7 \\
\hline $\begin{array}{c}\text { Useful but provision of alerts when group } \\
\text { members interact with environment would be } \\
\text { helpful }\end{array}$ & 2 \\
\hline Confusing to utilize certain features & 1 \\
\hline Very useful and the functionality is available & 7 \\
\hline Functionality available but is not utilized & 5 \\
\hline No Response & 3 \\
\hline
\end{tabular}

When asked about the usefulness of the system to collaborate with group members, most respondents said that it is easy to share files and communicate through group emails, discussion boards and wikis. Two respondents believed that the system lacks consistency per module, so some modules have the functionality available while others don't. Another 2 participants thought that the provision of alerts or announcements each time a member of the group makes changes or interacts with the system would be helpful.

\begin{tabular}{|c|c|}
\hline $\begin{array}{c}\text { Table 2 Responses to Question 10 } \\
\text { Useful }\end{array}$ & $\begin{array}{c}\text { No of } \\
\text { Respondents }\end{array}$ \\
\hline Discussion Boards & 6 \\
\hline Announcements & 3 \\
\hline Assignment submission & 4 \\
\hline Group tools, wiki, file sharing, emailing & 5 \\
\hline Modules content and information & 6 \\
\hline Feedback & 1 \\
\hline Not Useful & 2 \\
\hline Files cannot be viewed online, it needs downloading & 2 \\
\hline Extra information/irrelevant information & 2 \\
\hline Usability & 3 \\
\hline No Responses & 7 \\
\hline
\end{tabular}


Question 10: What features or functions of the system you think are useful/not useful?

The participants' responses to this question were discussion boards, announcements about the events and the ability to submit assignment along with group collaboration tools, modules content and feedback for assignment provided via the VLE are useful. The fact that files such as lecture slides cannot be viewed online and had to be downloaded and authenticated each time is inefficient. Some users also criticised the usability of the system and irrelevant information in modules content or links to irrelevant information.

Question 11: Are there any functions that you would like to be added to the system?

Table 3 illustrates the functionality that users would like to be added to the current VLE. Most participants stated instant messaging and calendar, however Blackboard Learn already has incorporated these functionalities. The reason might be that the users do not know about it or how to use it due to complicated design. Recorded lectures, online file storage and screen sharing functionality have also been mentioned as desired functions to be added to VLEs, which will be considered in the following prototype design of VLEs.

\begin{tabular}{|c|c|}
\hline \multicolumn{2}{|c|}{ Table 3 Responses to Question 11 } \\
$\begin{array}{c}\text { Fo of } \\
\text { Respondents }\end{array}$ \\
\hline Attendance Report & 1 \\
\hline Calendar & 3 \\
\hline Instant Messaging & 5 \\
\hline Online File Storage & 1 \\
\hline Recorded Lectures & 3 \\
\hline Screen Sharing Functionality & 1 \\
\hline Video Conferencing & 3 \\
\hline No & 7 \\
\hline No Reponses & 7 \\
\hline
\end{tabular}

\section{PROTOTYPE DESIGN}

The results of the questionnaire introduced in the last section have substantiated the existence of effective Virtual Learning Environments. The results also identified some functionalities that require redesigning and functionalities that users want to be integrated in current VLEs, including a more straightforward Help usability, calendar, recorded lectures, instant chat, video conferencing, collaborative file editing and online file storage. A low fidelity prototype approach was implemented to design the user Interface for the functionalities discussed above. The prototype was created using Microsoft Expression Blend.

Figure 9 illustrates the main page of the system that interconnects all features and functionalities. Some of the users complained about the complexity of the system design and the availability of irrelevant information. Our design is very neat: a search bar at the panel indicates that users can search through the content of the system to easily find materials. The calendar is designed on the front page to make it clear to users the availability of the feature. The announcements are displayed in the main section of the system interface. The system will also generate announcement, for instance when working in group the system will generate announcements such as changes made, files uploaded by group members etc.

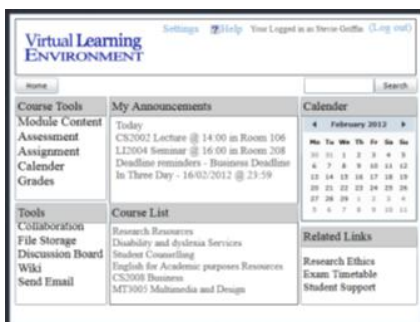

Figure 9 Main page of the prototype

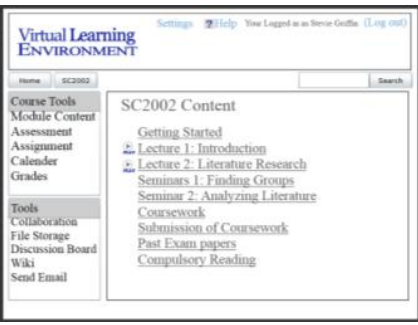

Figure 10 The module content
Figure 10 demonstrates the layout of the module content. The content of all the modules are searchable. The system provides a clear navigation and tells the user where they are. The play button next to the lecture slides indicates that the recording of the lecture is available. When clicking on the play button, a new screen will pop out to show a short introduction of what the lecture is about and the video of the lecture along with a short quiz.

Figure 11 illustrates the instant chat screen layout. On the right is the participants in the chat and the on the left the chat session. The participants can invite other people through the invite function, they can also find out more about the participants by clicking on the user info. The private chat will allow a user to chat to a selected user privately. The user also has the ability to share files. The leave chat button at the top right corner ends the session for the user.

Figure 12 demonstrates the video conferencing screen. The screens show the people in conference. The user can focus on a particular screen by clicking on it and it will be shown in the main window. The number of the screens will increase or decrease depending on the participants. All the functionality, such as voice, microphone and camera settings are available along with the ability to use keyboard to compose messages. The invite button on the top right corner indicates that user can invite other people. The Leave conference button will end the conference or log off the user.

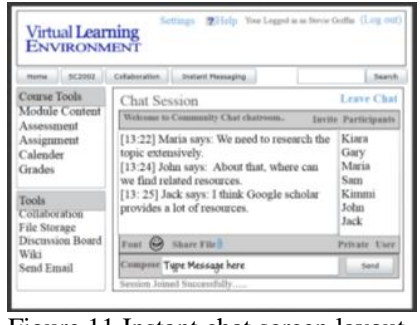

Figure 11 Instant chat screen layout Figure 12 Video conferencing screen 
Figure 13 demonstrates collaborative editing functionality. One of the users creates a document and the other users can edit it simultaneously. The text edited by different users is colour coded. The user is able to share the document with friends or colleagues or the public.

Figure 14 illustrates the online file storage functionality. The figure shows a simple interface where a list of the files uploaded by the user is displayed along with 'add' and 'delete' file functions.

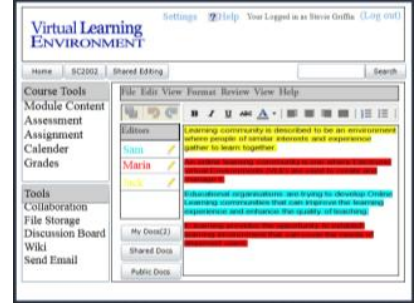

Figure 13 Collaborative editing

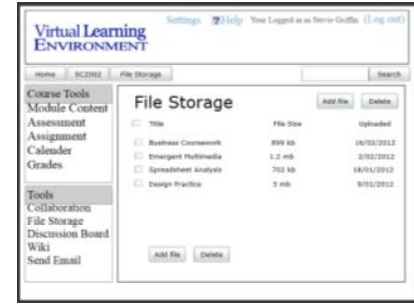

Figure 14 Online file storage
The prototype presented above suggests a design to simplify the functionalities of the tools provided by current VLEs and also to provide design recommendations for functionalities that are required by personal users. This prototype demonstrates a clear and neat layout. Although, not shown in the prototype but the system should have flexible and expandable architecture where features can be added and the functionalities of the system can be expanded.

\section{CONCLUSIONS AND FUTURE WORK}

The importance of collaborative learning for the effective performance of the students is indicated through the extensive research carried out in the field (Hunter, 2006). Virtual learning environments (VLEs) are nowadays widely employed to enhance a student's learning experience, by providing a set of teaching, learning and communication tools. These VLEs, however, are not fully used or exploited to support effective collaborative learning. This paper investigates the requirements and properties of VLEs for online collaborative learning, by examining existing learning platforms and exploring online collaborative learning characteristics. Questionnaire was distributed to students of Brunel University to investigate opinions and requirements from personal users. The results have showed that current VLEs such as Blackboard are mainly deemed useful but there are also a few functionalities that are not recognised by the students due to the complicated design. The questionnaires have also identified additional functionalities that can be integrated to the system to help improve students' learning. Based on the questionnaire results, a prototype has been designed to improve the current VLEs by simplifying some existing functionalities and incorporating extra functionalities required by the users. The prototype design has received positive feedback from the users questioned, for its straightforward, easy-to-use interface and added useful functions such as collaborative file editing and online file storage.

The work presented in this paper is an attempt to build effective collaborative virtual learning environments. Its investigation on existing VLEs and personnal users requirements and the corresponding prototype design provide useful suggestions for future VLE development. In the future, we will implement and test the prototype in practice to see how feasible and efficient it is and how to improve it to suit practical use. Moreover, we will inverstigate further the opinions and requirements from personal users, particularly with the use of novel technologies such as social websites and cloud computing, so as to develop advanced VLEs to suit changing user requirements.

\section{ACKNOWLEDGMENT}

The authors are grateful to the anonymous reviewers for their insightful comments and constructive suggestions.

\section{REFERENCES}

[1] E. Allen and J. Seaman, "Staying the course: Online Education in the United States", Needham, MA: Sloan Consortium (Sloan-C), 2008.

[2] E. Allen and J. Seaman, "Online Nation Five Years of Growth in Online Learning". The Sloan Consortium, pp.1, 2007.

[3] E. Allen and J. Seaman, "Sizing the Opportunity: The Quality and Extent of Online Education in the United States", Wellesley, MA: The Sloan Consortium, 2003.

[4] F. Bagherian and W. Thorngate, "Horses to water: Student use of course newsgroups", First Monday, 5(8), 2000.

[5] M. Belgrove, et al., "Using e-Learning Tools to Build a Community of Distance Learners: A Progress Review and Call for Collaboration", Proc. of the 7th European Conference on eLearning (ECEL 2008), Reading: Academic Publishing Limited, pp.90-96, 2008

[6] S. Chang, "Online Learning Communities with Online Mentors (OLCOM): A Model of Online Learning Communities", The Quarterly Review of Distance Educaiion, 5 (2), pp.75-88, 2004

[7] J. Mazak and F. Manista, "Collaborative Learning", The Reference Librarian, 32 (67-68), pp.225-242, 2000.

[8] Murdock, J. et al., "Creating an Online Learning Community: Is it Possible?", Innovative Higher Education, 36 (5), pp.305-315, 2001.

[9] D. Hunter, "Assessing collaborative learning", B. J. Music Ed, 23 (1), pp.75-89, 2006.

[10] A. Thurston, "Building Online Learning Communities", Technology, Pedagogy and Education, 14 (3), pp.353-370, 2005 